\title{
Long-term outcome after coronary artery bypass grafting in cardiogenic shock or cardiopulmonary resuscitation
}

\author{
Paul Sergeant, MD, $\mathrm{PhD}^{\mathrm{a}}$ \\ Bart Meyns, MD, PhD \\ Patrick Wouters, MD, PhD \\ Roland Demeyere, $\mathrm{MD}, \mathrm{PhD}^{\mathrm{b}}$ \\ Peter Lauwers, MD, PhD ${ }^{\mathrm{c}}$
}

\footnotetext{
From the Departments of Cardiac Surgery, ${ }^{a}$ Anaesthesiology, ${ }^{\mathrm{b}}$ and Intensive Care, ${ }^{\mathrm{c}}$ Gasthuisberg University Hospital, Katholieke Universiteit Leuven, Leuven, Belgium.
}

Read at the Eighty-second Annual Meeting of The American Association for Thoracic Surgery, Washington, DC, May 5-8, 2002.

Received for publication May 2, 2002; revisions requested June 14, 2002; revisions received April 25, 2003; accepted for publication June 6, 2003

Address for reprints: Paul Sergeant, MD, $\mathrm{PhD}$, Cardiac Surgery Department, Gasthuisberg University Hospital, Herestraat 44, B-3000 Leuven, Belgium (E-mail: Paul.Sergeant@uz.kuleuven.ac.be).

J Thorac Cardiovasc Surg 2003;126: 1279-87

Copyright $(92003$ by The American Association for Thoracic Surgery

0022-5223/2003\$30.00+0

doi:10.1016/S0022-5223(03)01289-3
Objectives: Coronary artery bypass grafting carried out in patients in cardiogenic shock or receiving cardiopulmonary resuscitation is an infrequently performed procedure, disrupting the normal process with a dramatic early risk. These circumstances mandate an analysis of the benefit, including the early identification of the late survivors.

Methods: A consecutive series of patients undergoing coronary artery bypass grafting while in cardiogenic shock $(n=167)$ or while receiving cardiopulmonary resuscitation $(\mathrm{n}=92)$ from August 1979 until August 2001 were studied by using time-related and multivariate methodologies and a common-closing-date follow-up methodology. The events leading to the preoperative condition were either a recent catheterization, recent coronary artery bypass grafting, recent percutaneous transluminal coronary angioplasty, an infarction at home, an infarction in the hospital, or an infarction after a recent infarction.

Results: The 1- and 10-year survival was $59 \% \pm 6 \%$ and $47 \% \pm 7 \%$, respectively. A normal hazard of late death was observed beyond the protracted early hazard. Multivariate analysis of survival identified an increased risk in the presence of additional comorbidity, treated diabetes, a lower $\mathrm{pH}$ at entry into the operating theater, and the presence of triple-vessel disease. The discriminatory power for hospital mortality of the predictive variables was low (receiving operator characteristic range, 0.56-0.69). The 30-day freedom from hospital discharge alive was $33 \% \pm 7 \%$. The 8-day freedom from stroke was $94 \% \pm 3 \%$, and 8-day freedom from mechanical univentricular or biventricular support was $87 \% \pm 5 \%$. The 10-year freedom from cardiosurgical reintervention was $90 \% \pm 6 \%$.

Conclusions: Coronary artery bypass grafting in cardiogenic shock or during cardiopulmonary resuscitation has an extremely high and protracted periprocedural risk but is balanced by a satisfactory late survival. An early prediction of the hospital survivors is difficult.

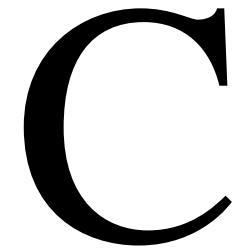
oronary artery bypass grafting $(\mathrm{CABG})$ has become one of the most routinely performed surgical procedures in the Western world. Patients in cardiogenic shock or receiving cardiopulmonary resuscitation (CPR) requiring immediate coronary surgery revascularization transgress this routine. These patients have a very disruptive effect on the organization of a CABG production line, not just because of the extreme urgency of their condition but also because of the highly specific experience required in the anesthetic and surgical management. In addition, they create the impression of gluttonous consumption of resources, with extremely low hospital survival and high morbidity. These procedures are therefore definitely at risk in health care environments in which cost reduction and fixed annual 
budgeting have become commonplace. The intuitive inference building on this subject is further aggravated by the complex variability in the clinical path after surgical intervention. This variability includes exuberant rates for revisions for bleeding, secondary closure of the sternum, placement and removal of an intra-aortic balloon pump (IABP) or univentricular and biventricular support devices, and sometimes even cardiac transplantation. This article analyses a single-center, 2-decade experience in this domain. The analysis moves away from the individual postoperative paths of these patients and focuses on the generation of data on the early and late risk or benefit. The use of hospital stay and assist devices is studied, as is the discriminatory power of some of the available variables in the prediction of the hospital survivors.

\section{Material and Methods}

A consecutive series of 259 patients undergoing coronary artery surgery revascularization in cardiogenic shock $(n=167)$ or during CPR $(\mathrm{n}=92)$ was analyzed and followed. The clinical status was classified at entry of the operating theater (CPR group) or at first hemodynamic evaluation in the operating theater (shock group), irrespective of the condition in the previous time interval. Patients in ventricular fibrillation but successfully defibrillated before entry to the operating theater were not classified as being in the CPR group.

Shock was defined as a cardiac index of less than $2 \mathrm{~L} \cdot \min ^{-1}$ - $\mathrm{m}^{-2}$ (despite pharmacologic support and optimization of cardiac loading conditions) in the presence of an acute ischemic event. Fixed electrocardiographic (ECG) findings unresponsive to therapy confirmed the presence of ischemia. The time interval between the ischemic event and the start of the operation should not have exceeded 24 hours. Patients with acute infarctions in stable hemodynamic condition were excluded from the study, as were patients requiring valvular, septal wall, or cardiac wall reconstruction.

The study inclusion interval started in August 1979 and closed in August 2001. All procedures were performed in a single institution (Gasthuisberg University Hospital, Leuven, Belgium) by a single group of surgeons and anesthesiologists. The mean number of cases per year was $11 \pm 6$ cases, a prevalence of $2 \%$ of our isolated coronary surgery activity. Events leading to or immediately preceding the clinical condition were coronary angiography $(20 \%)$, percutaneous coronary angioplasty (33\%), coronary bypass procedure $(9 \%)$, infarction at home $(17 \%)$, infarction in the hospital $(16 \%)$, or infarction after a recent infarction $(4 \%)$. The patients receiving CPR were operated on immediately, and the other patients were operated on within the first 2 hours after the event, with the first grafts open within 4 hours after the event in most patients.

The mean age of the patients in cardiogenic shock was $62 \pm 10$ years (range, 33-90 years); 50 patients were between 70 and 80 years of age, and 7 were older than 80 years. The mean age of the patients receiving CPR was $61 \pm 10$ years (range, 30-83 years). The female/male ratio was 28:72. Oral drug- and insulin-treated diabetes were observed in $10 \%$ of the patients. The extent of coronary artery disease was single-vessel disease in 14\%, 2-vessel disease in $28 \%$, and 3 -vessel disease in $58 \%$ of the patients. The current procedure was a redo CABG intervention in 39 patients. The EuroSCORE additive risk $^{1}$ of the combined dataset was $12.5 \% \pm 3 \%(12.3 \% \pm 3 \%$ for the shock dataset and $12.9 \% \pm 3 \%$ for the CPR dataset). The mean value for the first arterial $\mathrm{pH}$ after entry into the operating theater was $7.26 \pm 0.1$; it was $7.31 \pm 0.07$ for patients in the shock group (7 values missing) and $7.18 \pm 0.18$ for patients in the CPR group (5 values missing).

A previous history of stroke was present in 18 patients, and an internal carotid artery stenosis of greater than or equal to $80 \%$ was present in 12 patients. Severe atherosclerotic ascending aortic disease was identified in 13 patients.

\section{Anesthetic and Surgical Organization and Method}

The surgical organization started with the 24-hour/7-day onsite availability of a senior anesthesia supervisor and a cardiac surgery-experienced scrub nurse. The surgical and perfusion teams had a 24-hour/7-day call availability of less than 15 minutes.

On arrival in the operating theater, patients received a 5-lead ECG, pulse oximetry, an arterial pressure line, a large-bore peripheral and central venous line, and a thermodilution pulmonary artery catheter before (shock group) or after (CPR group) induction of anesthesia. Anesthesia consisted of etomidate, sufentanil (8-10 $\mu \mathrm{g} / \mathrm{kg}$ ), pancuronium, and midazolam (or propofol). Twodimensional transesophageal echocardiography was used in the majority of patients. Additional ECG leads were placed for IABP synchronization before draping. This management remained unchanged throughout the study interval.

The standard surgical approach for all first and all early repeat procedures, even in CPR, was median sternotomy, arterial and venous cannulation, cooling to $28^{\circ} \mathrm{C}$, and intravenous infusion of $1 \mathrm{mg} / \mathrm{kg}$ lidoflazine ${ }^{2}$ as myocardial protection. In all late redo procedures, femoral artery and vein cannulation was performed before sternotomy. The number of constructed grafts per patient was $2.1 \pm 0.8(2.2 \pm 0.8$ for the shock group and $2.0 \pm 0.8$ for the CPR group). The number of constructed distal anastomoses per patient was $2.5 \pm 1(2.6 \pm 1$ for the shock group and $2.4 \pm 1$ for the CPR group).

Arterial grafting was strongly influenced by the possible deterioration of the clinical condition, the urgency of the start of extracorporeal circulation, and the possible deleterious effects of shock on internal thoracic artery flow. ${ }^{3}$ In some selected very young patients, the arterial graft was prepared after the start of extracorporeal circulation. The number of constructed distal arterial anastomoses per patient was $0.4 \pm 0.6(0.5 \pm 0.6$ for the shock group and $0.1 \pm 0.4$ for the $\mathrm{CPR}$ group).

One-hour reperfusion was the standard practice from the earliest experience. IABPs, univentricular and biventricular assist devices, and occasionally heart transplantation were used when possible and deemed appropriate. Ventricular assist devices were used, since their availability in the late 1980 s, similar to the transplantation option.

\section{Variables, Events, and the Analytic Method}

The following continuous variables were considered: age, EuroSCORE risk value, $\mathrm{pH}$ at entry to the operating theater, number of distal anastomoses, number of grafts, number of arterial grafts, and date of operation. Transformation of variables was avoided because of the search for clinically understandable predictors. 
TABLE 1. The discriminatory power of 3 different continuous variables in the positive prediction of hospital mortality expressed as the area under the ROC curve

\begin{tabular}{|c|c|c|c|c|c|c|}
\hline Dataset & Variable & Area $\pm \mathrm{SE}$ & $\begin{array}{l}\text { Pivot } \\
\text { value }\end{array}$ & $\begin{array}{c}\text { Pivot } \\
\text { sensitivity }\end{array}$ & $\begin{array}{c}\text { Pivot } \\
\text { specificity }\end{array}$ & $\begin{array}{c}\text { Pivot }+ \text { predicted } \\
\text { value }\end{array}$ \\
\hline Combined & Age & $0.56 \pm 0.04$ & $>63 y$ & $58 \%$ & $57 \%$ & $42 \%$ \\
\hline Shock & Age & $0.56 \pm 0.05$ & $>62 y$ & $67 \%$ & $47 \%$ & $35 \%$ \\
\hline CPR & Age & $0.60 \pm 0.06$ & $>63 y$ & $60 \%$ & $64 \%$ & $56 \%$ \\
\hline Combined & Risk & $0.69 \pm 0.04$ & $>12 \%$ & $66 \%$ & $68 \%$ & $53 \%$ \\
\hline Shock & Risk & $0.69 \pm 0.05$ & $>11 \%$ & $77 \%$ & $55 \%$ & $43 \%$ \\
\hline CPR & Risk & $0.67 \pm 0.06$ & $>12 \%$ & $73 \%$ & $64 \%$ & $60 \%$ \\
\hline Combined & $\mathrm{pH}$ & $0.65 \pm 0.03$ & $\leq 7.27$ & $53 \%$ & $74 \%$ & $52 \%$ \\
\hline Shock & $\mathrm{pH}$ & $0.58 \pm 0.05$ & $\leq 7.27$ & $45 \%$ & $79 \%$ & $49 \%$ \\
\hline CPR & $\mathrm{pH}$ & $0.69 \pm 0.06$ & $\leq 7.11$ & $50 \%$ & $82 \%$ & $68 \%$ \\
\hline
\end{tabular}

The pivot value is given, as are the sensitivity, specificity, and positive predictive values of this pivot value. Age is the age at surgical intervention, risk is the EuroSCORE-predicted risk, and $\mathrm{pH}$ is the first $\mathrm{pH}$ at entry to the operating theater.

The following nominal variables were considered: sex, surgeon, vessel disease, diabetes and form of therapy, redo procedure, presence of severe internal carotid disease $(\geq 80 \%)$, presence of severe ascending aortic disease, history of stroke, and reason for clinical state.

The cardiac and noncardiac variability was described by using the additive EuroSCORE-predicted risk value. The $\mathrm{pH}$ at entry to the operating theater was the first arterial $\mathrm{pH}$ value taken after entry to the operating theater.

A common-closing-date methodology was used to complete the follow-up of the hospital survivors, even though most clinical information was already stored in the K.U. Leuven Coronary Surgery Database. The common closing date was September 1, 2001. The follow-up was complete for all patients. The events after the operation were presented in a time-related manner ${ }^{4}$ and analyzed accordingly in a multivariate manner (when appropriate).

Hospital stay included the complete interval of hospital stay in the primary or secondary hospital, as well as the stay in the rehabilitation or coma center. The hospital stay observations were extremely skewed and competed strongly with early mortality, thereby forcing the use of time-related depictions and analyses. The patients for the event "hospital stay" were censored at death or at the common closing date.

The patients for the event "freedom from death" were censored at the common closing date or at the end of incomplete follow-up. The patients for the event "freedom from decerebration or stroke" and the event "freedom from mechanical univentricular or biventricular support" were censored at death (if before day 8 after the operation) or at day 8.

A cardiosurgical reintervention was defined as any cardiosurgical procedure involving a direct and open reconstruction of the heart or the ascending aorta; percutaneous transluminal coronary angioplasty procedures were excluded from the event. The patients for the event "freedom from cardiosurgical reintervention" were censored at death, the common closing date, or the end of incomplete follow-up.

A receiver operating characteristic curve (ROC) $)^{5,6}$ was used to evaluate the predictive performance (discriminatory power) of some continuous variables (Table 1).
The maximum discriminatory power of the continuous variable (pivot value) in the prediction of hospital mortality was identified as the point at which the rate of change of the tangent to the curve is maximal, the place where the least false-negative and falsepositive results were observed. This was calculated for the complete dataset and for the 2 subgroups.

Cox proportional hazard methodology was used for hospital stay and survival. The goodness of fit of these 2 final models was examined by plotting the martingale residuals against the fitted value of the linear predictors for each case in the dataset and by plotting the deviance residuals.

\section{Results}

\section{Hospital Mortality}

The hospital mortality was 35\% (30\%-42\% values for the $95 \%$ confidence limits) in the complete dataset, $30 \%$ (23\%$37 \%$ values for the $95 \%$ confidence limits) in the shock subgroup, and 43\% (33\%-54\% values for the 95\% confidence limits) in the CPR subgroup.

The difference between the additive EuroSCORE-predicted risk and the observed mortality was important for the complete dataset $(12.5 \%$ vs $35 \%, P<.0001)$, as well as for the shock $(12.3 \%$ vs $30 \%, P<.0001)$ and CPR subgroups (12.9\% vs $43 \%, P<.0001)$.

A logistic regression analysis of hospital mortality identified the first arterial $\mathrm{pH}$ at entry in the operating theater (lower value equaled worse hospital mortality, $P=.001$ ) and the EuroSCORE risk (higher value equaled worse hospital mortality, $P<.0001)$ as independent predictors. The $R^{2}$ value was 0.11 . Table 1 lists the discriminatory analysis of some selected continuous isolated and combined variables on the prediction of hospital mortality. Figure 1 depicts the ROC analysis and the distribution of the values for the variable "pH at entry" on hospital survival.

In the interval between January 1997 and January 2001, the shock and CPR groups represented $2.1 \%$ of the isolated 

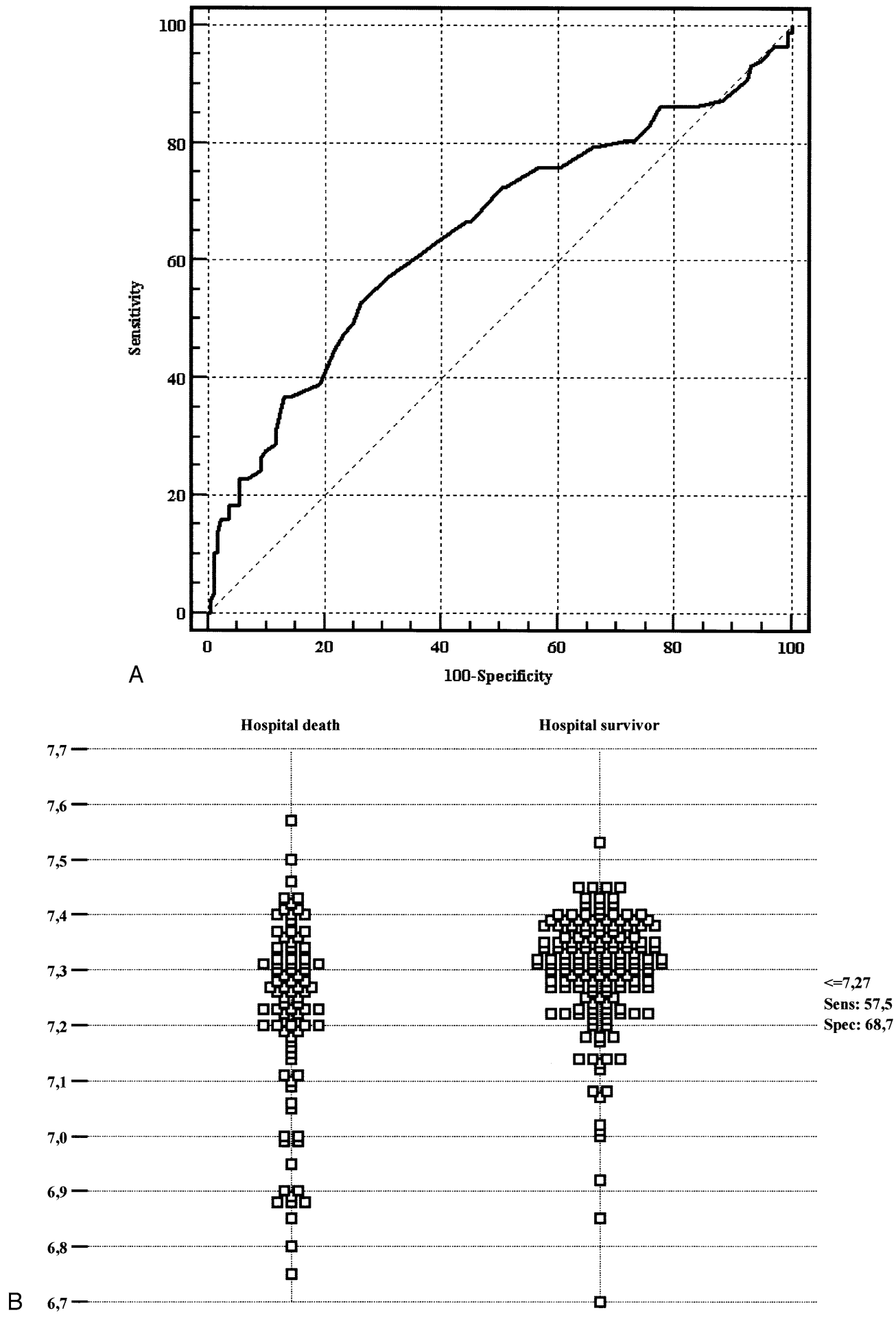

Figure 1. The ROC analysis of the discriminatory power of "pH at entry" on hospital mortality is shown in A. The sensitivity is plotted versus the $\mathbf{1 0 0}$ - specificity of the test, and the points are interconnected. The area under the thick line is the measure of the discriminatory power. B explores the distribution of the values for the hospital survivors and nonsurvivors and the pivot value. 


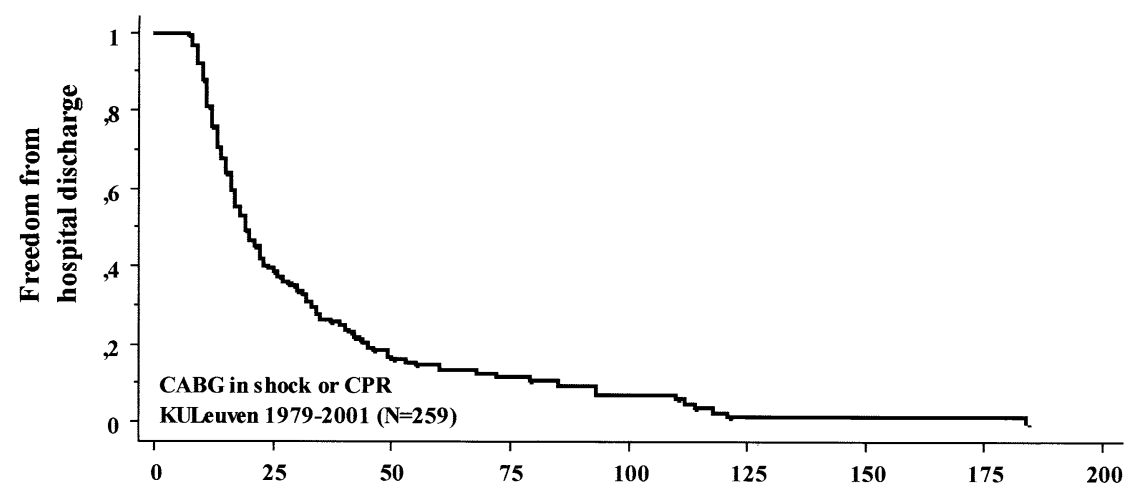

A

Days

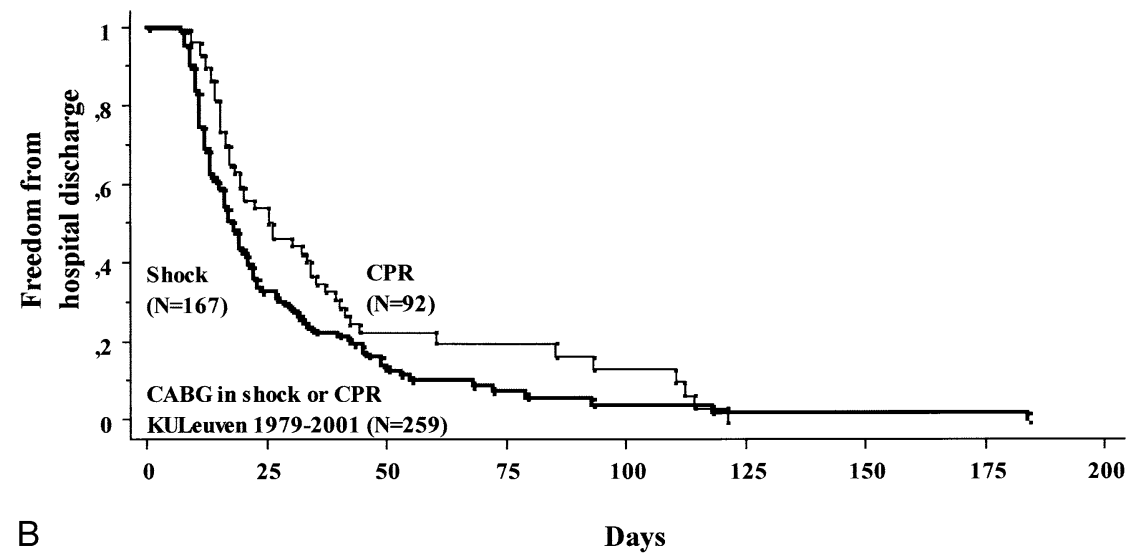

Figure 2. The Kaplan-Meier time-related depiction of the freedom from hospital discharge alive: A, the total group; $\mathrm{B}$, the 2 subgroups. The thick line represents the shock subgroup, and the thin line represents the CPR subgroup.

CABG population at our institution and $20.2 \%$ of the total hospital mortality for isolated CABG.

\section{Hospital Stay}

The 10-day, 20-day, and 30-day freedoms from hospital discharge alive (Figure 2, A) were, respectively, 88.0\% \pm $5 \%, 46.8 \% \pm 6 \%$, and $33.5 \% \pm 7 \%$ for the complete dataset. The 30-day freedoms from hospital discharge alive were $28.1 \% \pm 8 \%$ for the shock dataset (Figure $2, B$ ) and $44.7 \% \pm 13 \%$ for the CPR dataset (Figure 2, $B$ ). The mean hospital stay of the hospital survivors was $26 \pm 26$ days overall, with a median value of 17 days and a mode of 11 days. The skewness of the observations was 2.9.

A Cox proportional hazard, multivariate, time-related model for hospital stay identified an incremental risk in the EuroSCORE-predicted risk for hospital mortality (higher value equaled longer stay, $P=.008$ ), the presence of single-vessel disease (presence equaled shorter stay, $P=$ .003 ), the presence of drug-treated diabetes (presence equaled longer stay, $P=.03$ ), and the date of the operation (more recent date equaled longer stay, $P=.03$ ).
In the interval between January 1997 and January 2001, the shock and CPR groups represented $2.1 \%$ of the isolated CABG population at our institution and used $4.6 \%$ of the total hospital stay for isolated CABG (discharge alive and discharge dead).

\section{Survival}

The 1-year, 10-year, and 15-year survivals (death by any cause anytime after the operation) for the complete dataset (Figure 3, A) were, respectively, 59.4\% $\pm 6 \%, 47.5 \% \pm$ $7 \%$, and $40.6 \% \pm 8 \%$. There were 81 patients at risk at 10 years and 13 at 15 years.

The 10-year survival was $53.2 \% \pm 8 \%$ with 60 patients at risk for the shock dataset (Figure 3, B) and 36.9\% $\pm 10 \%$ with 22 patients at risk for the CPR dataset (Figure 3, B). The early risk of dying stabilized between the 50th (shock group) and 100th day after the operation (CPR group).

A Cox proportional hazard, multivariate, time-related model for survival identified an incremental risk in the EuroSCORE-predicted risk for hospital mortality (higher value equaled worse survival, $P<.0001$ ), the extent of the 


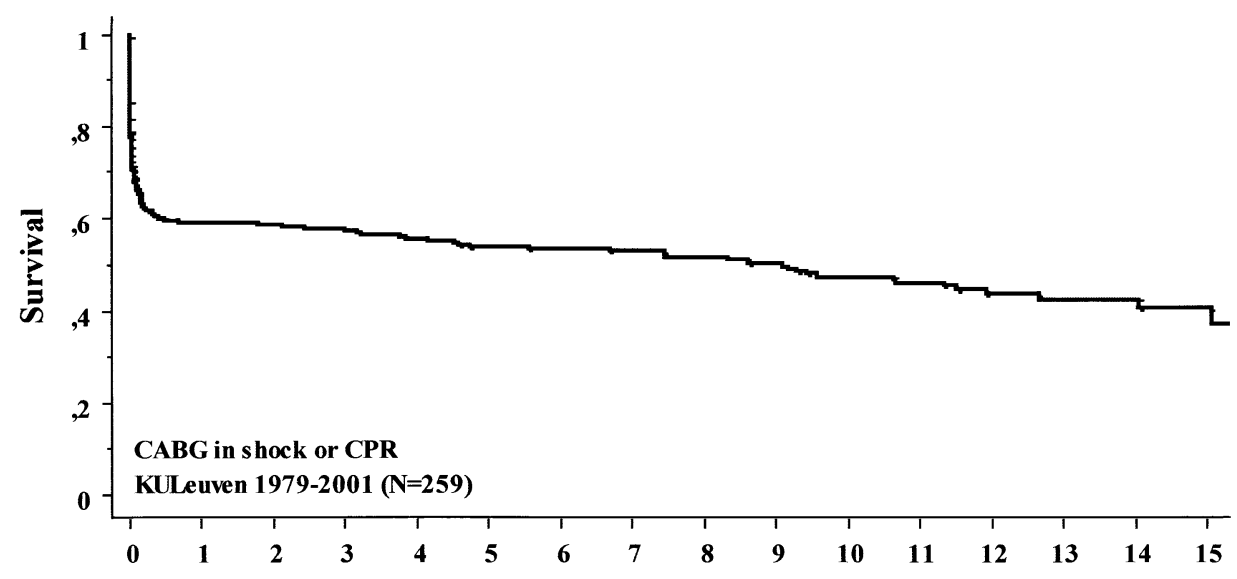

A

Years

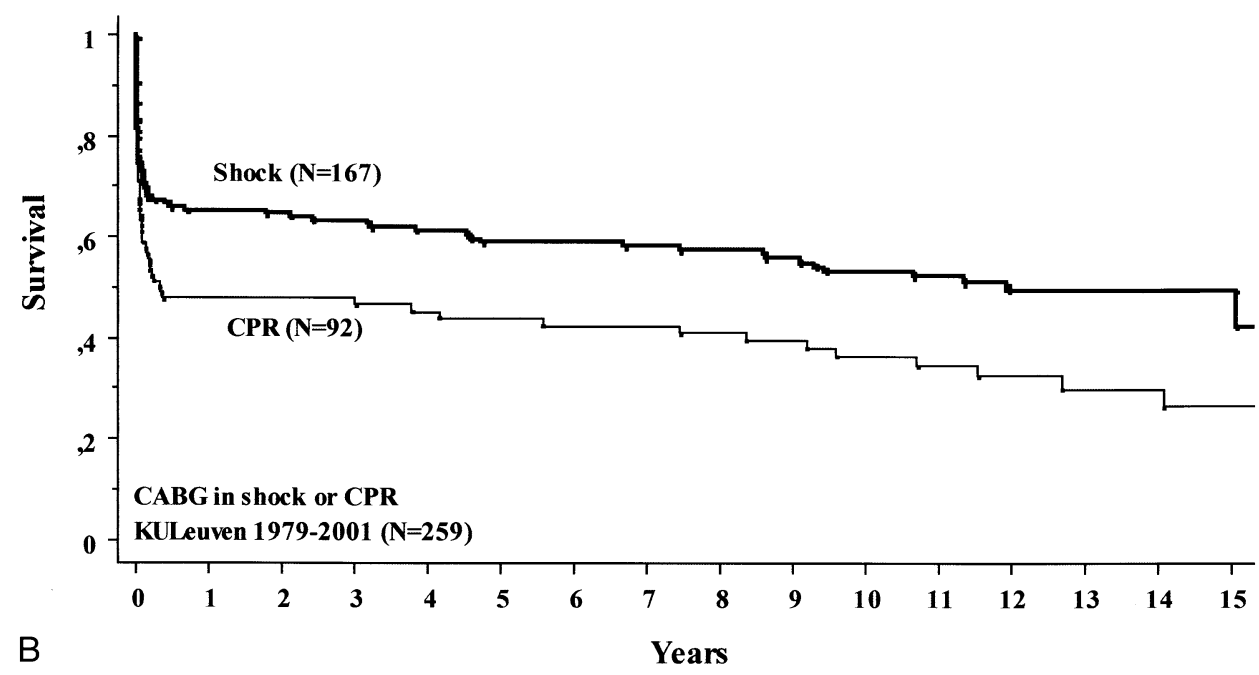

Figure 3. The Kaplan-Meier time-related depiction of the freedom from death: A, the total group; B, the 2 subgroups. The thick line represents the shock subgroup, and the thin line represents the CPR subgroup.

coronary disease (3-vessel disease equaled worse survival, $P=.01$ ), the presence of drug-treated diabetes (presence equaled worse survival, $P=.001$ ), and the arterial $\mathrm{pH}$ at entry to the operating theater (lower value equaled worse survival, $P<.0001)$.

\section{Other Events}

Hemodialysis or continuous hemofiltration was used in 37 (14\%) patients. An IABP was present or inserted in 104 (40\%) patients.

The 8-day freedom from decerebration or stroke after CABG was $94.1 \% \pm 3 \%$ for the complete dataset $(97.6 \% \pm$ $2 \%$ for the shock subgroup and $87.7 \% \pm 7 \%$ for the CPR subgroup). A Cox proportional hazard, multivariate, timerelated model identified an incremental risk in a previous history of stroke (history equaled higher risk, $P=.002$ ) and the $\mathrm{pH}$ value at entry in the operating theater (lower value equaled higher risk, $P<.0001$ ).

The 8-day freedom from mechanical univentricular or biventricular support was $87.4 \% \pm 5 \%$.

The 1-year and 10-year freedoms from cardiosurgical reintervention were $97.9 \% \pm 2 \%$ and $90.1 \% \pm 6 \%$, respectively. There were 3 transplantations (days 2, 9, and 2018), 10 redo CABGs (days 3, 1151, 1616, 1670, 3129, 3132, $3367,3483,3792$, and 3819), 2 aortic valve replacements (days 4291 and 4952), and 1 repair of a false aneurysm of the aorta (day 32).

\section{Discussion}

Limitations of the Dataset, Variables, and Analysis Isolated primary or repeat $\mathrm{CABG}$ in patients in cardiogenic shock and those receiving CPR is a rare procedure, even in 
centers organized for this approach on an around-the-clock basis. ${ }^{7}$ The validity of this dataset is reduced through a bias in patient selection. Not all patients with similar events after a coronary angiography, a percutaneous coronary angioplasty, an infarction at home, an infarction in the hospital, or an infarction after a recent infarction were presented for surgical intervention. On the contrary, most patients with this condition after coronary bypass surgery were treated surgically and therefore included.

A grouped variable, the EuroSCORE, enhances the possibility of a stable multivariate analysis. A stable coefficient for one of the composing elements of this score would be very unlikely because of the limited sample size of the dataset. The EuroSCORE is a recent (1999) scoring system and has been applied retroactively on a historical dataset; this is somewhat unfair for this system.

The sample size remains still limited for complex mathematic modeling; extending the inclusion interval would probably induce time strata in the analysis. An additional possible weakness is certainly the continuous improvements in patient management. Aggregate data covering this interval might not be relevant for clinicians facing these issues today. The effect of the time axis was analyzed for every event. In our institution these changes have been less than in some other places. The general immediate management has remained unchanged. The extensive use of support systems has always been similar, whether using intracorporeal or extracorporeal support systems from company $\mathrm{X}$ in the 1980s or 1990s and from company Y in the current era.

The primary variable, CPR or shock, carries a possible bias. The low value for the variable "pH at entry" in some patients in the shock group is a clear indication that these patients could have benefited from CPR. The forced use of strata in the analysis has therefore been avoided. Validation of the mathematic models with a study and test sample would have further decreased the sample sizes.

\section{Early Events Versus Late Survival}

The very high hospital mortality is a dramatic observation strongly influenced in both directions by the inclusion criteria and bias. Very little structured evidence is available in the literature on the surgical therapy of patients requiring CPR. The landmark SHOCK trial has probably the best available information concerning alternative approaches in the therapy of cardiogenic shock. The inclusion criteria were different from those of the present study and included only postinfarction patients. Hochman and colleagues ${ }^{8}$ reported an overall in-hospital mortality of $66 \%$, uncorrected for therapy, in a SHOCK pretrial study. In the actual SHOCK trial ${ }^{9} 30$-day mortalities of $47 \%$ and $56 \%$ were recorded, respectively, for the interventional and medical therapies of myocardial infarction complicated by cardiogenic shock. Our evidence suggests that an observation interval of 30 days is inappropriate for the analysis of the in-hospital or early mortality. The 6-month mortality was lower for the revascularization alternative, with $50 \%$ for the interventional approach and $63 \%$ for the medical therapy.

Health care purchasers and, even more, the actual surgical teams tackling this clinical entity of shock and CPR are most interested in the discriminatory ability of predicting the hospital survival before or early in the procedure. Often commonly available variables and pivot values have been used without knowledge of their power. The SHOCK pretrial report authors were disappointed that readily available clinical variables had limited predictive power. The unreliable discriminatory effect on survival of base excess, $\mathrm{pH}$, or potassium levels was similarly identified in a population ${ }^{10}$ with severe hypothermia caused by cold-water submersion and cardiac arrest.

The logistic model of hospital mortality in this analysis explained only $11 \%$ of the variability. The very disappointing values of the area under the ROC curves for the clinical variables were a similar frustration. The very low sensitivity, specificity, and positive predictive power of the pivot values do not allow their clinical use. The reasonable result with "the first $\mathrm{pH}$ " for the patients receiving CPR probably suggests a search in the direction of biologic discriminatory markers representing the deterioration of the overall condition and the severity and duration of the (often poorly documented) preceding shock episode.

The failure of the additive EuroSCORE in the actual prediction of hospital mortality has important consequences for the inclusion of these patients in quality audits, using this system for correction of patient variability. This failure of scoring systems in high-risk patients was observed ${ }^{11}$ earlier in a large recent cohort of patients undergoing CABG with a EuroSCORE value of greater than $11 \%$, including patients without infarction. A neutralization of the patients in the shock and CPR CABG groups in an interdepartmental audit is probably appropriate. These mortalities represented $20 \%$ of the departmental mortality in patients undergoing $\mathrm{CABG}$ in the same time frame.

The very strong power of mortality competes with all nonlethal events, such as hospital stay, stroke, mechanical support, hemodialysis, and late reintervention. If the early survival in these patients could be improved, a totally different outcome in these nonlethal events would be observed. Even under these limitations it remains interesting that only $13 \%$ of the patients had irreversible brain damage through their resuscitation and surgical process.

It is difficult to evaluate the quality of the performed reconstruction similarly in competition with the extreme early mortality. Two events are appropriate: the survival beyond the early phase and the freedom from surgical reintervention. The cumulative survival hazard of CABG in patients with shock and those receiving CPR flattened com- 
pletely beyond 100 days after the operation. The freedom from surgical reintervention was very acceptable.

\section{Resource Consumption}

Different types of resources, both human and material, are used in treating patients undergoing $\mathrm{CABG}$ in cardiogenic shock and during CPR. The consequences of treating these patients will differ considerably between health care economic systems. In some countries these will be just additional charges, whereas in others these will be unrecoverable costs. The most visible resource consumption, hospital stay, was only doubled in these patients. This event was once more influenced by the competing early hospital mortality. A higher price was paid for the use of hemodialysis and support devices. Some survivors with stroke have been confronted with the medical and socioeconomic consequences of this condition and the partly failed therapy.

Our departmental policy has changed to mandating an immediate surgical approach consisting of initiation of extracorporeal circulation for all patients in cardiogenic shock or receiving CPR and amendable by CABG. If the first arterial $\mathrm{pH}$ is less than 6.9, we advise discontinuation of all therapy and urge the caregiver to proceed with extreme caution if the $\mathrm{pH}$ is less than 7.1. The use of additional resources (dialysis, intermediary, and long-term mechanical support) is weighed against the life expectancy (judged by the presence and severity of comorbidity).

Patients undergoing CABG in cardiogenic shock and during CPR have disrupted the surgical routine of coronary surgery. They have induced an additional opportunistic resource cost.

The appropriate use of health care resources in a population is a delicate balance between an individual patient's rights and the collective responsibility of the community. This study suggests that health care purchasers should allow and cover this approach. Clinicians should continue the search for clinical discriminative markers guided but not driven by available variables.

\section{References}

1. Nashef S, Roques F, Michel P, Gauducheau E, Lemeshow S, Salamon $\mathrm{R}$, et al. European system for cardiac operative risk evaluation (EuroSCORE). Eur J Cardiothorac Surg. 1999;16:9-13.

2. Flameng W, Daenen W, Borgers M, Thoné F, Xhonneux R, Van de Water A, et al. Cardioprotective effects of lidoflazine during 1 hour normothermic global ischaemia. Circulation. 1981;64:796-807.

3. von Segesser LK, Lehmann K, Turina M. Deleterious effect of shock in internal mammary artery anastomoses. Ann Thorac Surg. 1989;47: 575-9.

4. Kaplan E, Meier P. Non-parametric estimation from incomplete observations. J Am Stat Assoc. 1958;53:457-81.

5. Zweig M, Campbell G. Receiver-operating characteristic (ROC) plots: a fundamental evaluation tool in clinical medicine. Clin Chem. 1993; 39:561-577.

6. Swets J. Measuring the accuracy of diagnostic systems. Science. 1988;240:1285-93.

7. Sergeant P, Blackstone E, Meyns B. Early and late outcome after
CABG in patients with evolving myocardial infarction. Eur J Cardiothorac Surg. 1997;11:848-56.

8. Hochman JS, Boland J, Sleeper LA, Porway M, Brinker J, Col J, et al. Current spectrum of cardiogenic shock and effect of early revascularisation on mortality. Circulation. 1995;91(3):873-81.

9. Hochman JS, Sleeper LA, Webb JG, Santorn TA, White HD, Talley $\mathrm{JD}$, et al. Early revascularisation in acute myocardial infarction complicated by cardiogenic shock. N Engl J Med. 1999;341(9):625-34.

10. Wollenek G, Honarwar N, Golej J, Marx M. Cold water submersion and cardiac arrest in treatment of severe hypothermia with cardiopulmonary bypass. Resuscitation. 2002;52(3):255-63.

11. Sergeant P, de Worm E, Meyns B. Single centre, single domain validation of the EuroSCORE on a consecutive sample of primary and repeat CABG. Eur J Cardiothorac Surg. 2001;20:1176-82.

\section{Discussion}

Dr Ludwig K. von Segesser (Lausanne, Switzerland). Given the difficult set of patients analyzed, a hospital mortality rate of $35 \%$ and an almost $50 \%$ survival after 10 years of follow-up is excellent. It comes as no surprise that early mortality is the main cause of loss of life and that patients requiring CPR do less well than patients with shock not requiring CPR.

Dr Sergeant, the observation period of this study is about 22 years, and therefore the mean number of patients operated on per year for shock is about 8 and for CPR is about 4 . It would be very interesting for the audience to know whether the distribution over time of the patients operated on is uniform. I am asking this because in our own experience, like in yours, the main cause of acute need of surgical intervention is an incident in the catheterization laboratory, for example, a crashed percutaneous transluminal coronary angioplasty. However, these events are unevenly distributed over time and much less frequent nowadays. If you get called in now for such patients, the myocardium has usually suffered already for a couple of hours, and all sorts of drugs, devices, and time have been wasted. Hence the patients we saw 20 years ago and those we see today are not exactly the same.

Furthermore, I would like to ask you to give us some insight in the delays observed between the initial event and the decision to go for surgical intervention, initiation of cardiopulmonary bypass, and delay until reperfusion. Is there a correlation between the time elapsed and outcome?

Dr Sergeant. Thank you, Professor von Segesser, for these questions. The first question is the prevalence or the incidence of these patients. Indeed, as you point out correctly, the prevalence is around 12 patients a year. You are correct that the patient's clinical condition might have changed over time. This becomes obvious on the basis of the importance of the biologic markers of " $\mathrm{pH}$ at entry of the operating theater" versus the cause leading to the operation.

Concerning the delay between the events: we have a system with 24-7 standby, basically on an around-the-clock basis. If our support is called in, we can be in 15 minutes on extracorporeal circulation around the clock. Therefore the delays are very short, but as you point out, the cardiologists and all our colleagues from different departments have spent a lot more time and energy in treating these patients. We have tried to build inferences from the chaotic clinical paths in all these different patients and bring this down in a more structured form.

Dr von Segesser. I had one last question to the expert in statistics you are. You write that a higher EuroSCORE is an independent predictor of mortality; however, EuroSCORE is the sum of semicontinuous and categoric variables having an arbitrary 